Conclusion The interim audit has shown that we have already made one HDU bed available for other patients for the equivalent of 2 months during the busiest period of the year when critical care beds are at crisis point. There were no adverse events during this new initiative and the median length of hospital stay for this patient group was not increased.

\section{GP279 A REVIEW OF THE DIAGNOSTIC EVALUATION OF COMPLICATED PARAPNEUMONIC EFFUSION OR EMPYEMA IN AN IRISH TERTIARY HOSPITAL}

'Oksana Kozdoba*, 'Patrick Gavin, ${ }^{2}$ Richard Drew, ${ }^{1}$ Des Cox. 'Our Ladys Children's Hospital Crumlin, Dublin, Ireland; ${ }^{2}$ Irish Meningitis ans Sepsis Reference Laboratory Temple Street Childrens University Hospital, Dublin, Ireland

\subsection{6/archdischild-2019-epa.338}

Introduction Complicated parapneumonic effusion or empyema is a relatively common complication of pneumonia, often requiring thoracocentesis. The diagnostic yield with traditional culture of blood or pleural aspirate specimens is low, emphesizing the role for new molecular techniques to improve identification of the responsible pathogens.

Aim The purpose of this study was to review the laboratory investigation of childhood complicated parapneumonic effusion or empyema with the view to optimising diagnosis.

Methods A retrospective review of paediatric cases of complicated parapneumonic effusion or empyema requiring thoracocentesis was undertaken in an acute tertiary referral paediatric hospital, over a five year period, from January 2014 to December 2018. Cases with clinical and radiographic findings consistent with a diagnosis of complicated parapneumonic effusion or empyema were only included if a sterile site specimen was taken for diagnostic microbiologic evaluation. Baseline patient demographic data, clinical findings, laboratory indices, microbiology results and imaging findings were collected.

Results Sterile site specimens from 43 children with parapneumonic effusion/empyema were identified (females,60\%). $79 \%$ of the children were younger then 5 years of age. $45 \%$ (14 of 31 ) of children who had virologic testing performed had at least one respiratory virus detected. Six children had multiple viruses detected. A causative bacteria was identified in 24 cases $(56 \%), 6$ by conventional culture (pleural fluid, 5; blood,1) and 21 by PCR (pleural fluid, 20; blood, 3). Three children had both culture and PCR positive. PCR had the highest detection rate of causative organism: pleural fluid PCR positive, $52 \%$ (20 of 38 tested); blood PCR positive, 50\% (3 of 6 tested). Pleural fluid culture positive, $11.6 \%$ (5 of 43 tested); and blood culture positive $2.5 \%$ (1 of 39 tested). Streptococcus pneumonia was the causative organism detected in $95 \%$ cases.

Conclusion This retrospective review confirms that in paediatric cases of complicated parapneumonic effusion or empyema traditional microbiological culture of sterile site specimens infrequently identifies a causative organism. For such culture negative cases, appropriate PCR testing significantly improves the detection rate of causative organisms.

\section{GP280 SLEEP DISORDERED BREATHING AND SPINA BIFIDA - EXPERIENCE IN A TERTIARY REFERRAL PAEDIATRIC CENTRE}

Aoiibhinn Walsh*, Angela Mernagh, John Caird, Darach Crimmins, Fiona Ringholz, Michael Williamson, Fiona Healy. Temple Street Children's University Hospital, Dublin, Ireland

10.1136/archdischild-2019-epa.339

Introduction There is a paucity of knowledge regarding Sleep Disordered Breathing (SDB) in paediatric patients with Spina Bifida/Myelomeningocoele (SB/MMC). Undiagnosed, untreated SDB has detrimental effects on growth, development (1) and cognition (2) which is especially important to recognise in those with other co-morbidities as is the case in $\mathrm{SB} / \mathrm{MMC}$.

Aim There are currently 317 patients aged 0-16 years attending the Spina Bifida service at Temple Street Children's University (TSCUH). Fourteen of 317 patients (4.4\%) currently require Non-Invasive Ventilation (NIV) therapy for SDB under the care of the Respiratory Team. We hypothesize that a subpopulation of the SB/MMC patients are at risk of undiagnosed SDB. We aimed to initially review the demographics of the sub-group of SB/MMC patients currently requiring NIV by assessing for potential predictors of SDB.

Method We used up to date medical records of the patients under our care to look for potential predictors for SDB, in particular hydrocephalus with VP shunt, Chiari II malformation and intellectual disability (ID). We then compared our data to what is known about the general SB/MMC population.

Results $100 \%$ of SB patients with SDB requiring NIV had hydrocephalus with VP shunt compared to $80 \%$ in general SB/MMC patients. 92\% also had Chiari II malformation. 50\% had a moderate to severe ID in contrast to $25-25 \%$ of the general SB/MMC population.

Conclusion A higher proportion of SB/MMC patients known to have SDB have hydrocephalus and ID compared to the SB/ MMC population mean. At present there is no standardised screen for SDB in our SB/MMC patients. The results of this review have prompted a clinical audit of all of SB/MMC patients at TSCUH to identify those at higher risk of SDB and ultimately determine the prevalence of SDB in this population.

\section{REFERENCES}

1. 'How do we recognize the child with OSAS?' Joosten KF, et al. Pediatric Pulmonology 2017 Feb;52(2):260-271.

2. 'Association of Sleep-Disordered Breathing With Cognitive Function and Risk of Cognitive Impairment: A Systematic Review and Meta-analysis.' Leng Y, et al. JAMA Neurology 2017 Oct 1;74(10):1237-1245. 